\title{
Utility of Hematological Scoring System in Diagnosis of Neonatal Sepsis
}

\author{
Aparna S ${ }^{1 *}$, Vivek George ${ }^{2}$, Limi Mohandas ${ }^{2}$, Lisha S Raj ${ }^{3}$ and Sheela Vasudevan² \\ ${ }^{1}$ SRRI, Trivandrum, India \\ ${ }^{2} \mathrm{SGMC}$, Trivandrum, India \\ ${ }^{3} \mathrm{KIMS}$, Trivandrum India,
}

\begin{abstract}
Background: Neonatal septicemia is defined as a clinical syndrome characterized by systemic signs and symptoms caused by a bacterial infection and gives positive blood culture in the first month of life. It is associated with high morbidity and mortality, but early diagnosis and treatment significantly improve the outcomes. The present study brings out a quick and cost- effective Hematological Scoring System that enables early diagnosis of neonatal sepsis
\end{abstract}

Method: This study was conducted in the Department of Pathology of Sree Gokulam Medical College and Research Foundation, Venjaramoodu, Thiruvananthapuram over 1 year. Eighty neonates with clinical suspicion of sepsis were studied with respect to their peripheral smear findings, blood culture and C-reactive protein levels.

Result: Among the eighty cases, twelve neonates were culture positive. Male gender and late- onset of sepsis were the significant risk parameters. Escherichia coli and Staphylococcus aureus were the most common isolated organisms. Of the different parameters studied I:T ratio and Absolute neutrophil count showed the highest specificity and immature neutrophil count had the highest sensitivity. Forty-one percent of neonates had a high Hematological Scoring System score. The specificity of Hematological Scoring System with a score $>5$ was $92 \%$

Conclusion: Hematological Scoring System is a cost-effective, rapid and easy to perform screening test that can be used to rule out sepsis thus avoiding unnecessary administration of antibiotics to unaffected babies. It should be adopted as a routine screening procedure by minimally qualified rural doctors with minimal resources to ensure appropriate action immediately for children with high index of suspicion of sepsis.

Keywords: Blood Culture, WBC Count, Early Onset Sepsis (EOS), I/T Ratio

\section{Introduction}

Neonatal septicaemia is defined as a clinical syndrome characterized by systemic signs and symptoms due to generalized bacterial infection with positive blood culture in the first four weeks of life. ${ }^{[1]}$ Accurate clinical diagnosis of neonatal septicaemia is an involved task. The early signs and symptoms are non-specific; however, treatment has to be started immediately, an outcome of septicaemia in neonate largely depends on its early identification and treatment ${ }^{[2]}$. Although blood culture is considered to be the gold standard for diagnosis, the inherent requirement of sophisticated laboratory equipment makes it less available for resource-poor countries. In addition, blood culture requires a minimum of 48-72 hours and yields positive results in only $30-40 \%$ of cases. ${ }^{[3,4]}$ Also the inability to isolate a microbial pathogen does not exclude sepsis ${ }^{[5]}$. Therefore, the necessity for a rapid and cost-effective test, with minimal time consumption is prevalent. An ideal diagnostic test for neonatal sepsis should have maximum sensitivity and specificity. In recent years, various investigators have evaluated some highly sensitive and specific inflammatory markers to diagnose neonatal sepsis. Although these markers are sensitive and specific, they also require sophisticated and expensive methodologies thereby making it impractical for developing countries. ${ }^{[6]}$

A comprehensive study of hematological parameters together as a combination of tests provided early predictions of neonatal septicemia. This combination study resulted in both increased sensitivity and specificity, thereby aiding in early treatment with appropriate antibiotics. ${ }^{[7]}$ Rodwell et. al. developed a seven-point hematologic scoring system based on the WBC count, total and immature neutrophil counts and ratios, degenerative changes in neutrophils and thrombocytopenia. This approach proved to be effective with $96 \%$ sensitivity and $99 \%$ negative predictive value was obtained. ${ }^{[8]}$

The present study evaluated the effectiveness of haematological profile in early diagnosis of neonatal sepsis.

\section{Materials and Methods}

This was a prospective cross-sectional study conducted in the Department of Pathology, over a period of 1 year from 
November 2014 to November 2015 with prior approval from institutional Ethical committee. Neonates up to thirty days of age were enrolled in the study if there were clinical suspicion of sepsis or any potential risk factors for sepsis like prematurity or prolonged rupture of membrane included in the study. Peripheral smear from severely jaundiced neonates, due to blood group incompatibilities, were not included in the study. Blood sample $(0.5-1.0$ $\mathrm{ml}$ ) obtained by peripheral venipuncture was collected in EDTA vial. A good quality peripheral smear prepared within 1 hour of venepuncture, stained with Leishman and Giemsa stains were examined under oil immersion with 100x magnification. In addition total leukocyte and platelet counts were counted by ADVIA 2120i automated system. Differential leukocyte counts were performed on Leishman and Giemsa stained blood smears by counting 100 cells. Immature neutrophils (promyelocyte, myelocyte, metamyelocyte and band forms) and degenerative morphological changes in neutrophils including toxic granulations, vacuolations and Dohle bodies were noted. The hematological findings were analysed according to HSS of Rodwell et al. HSS assigns a score of 1 for each of seven findings significantly associated with sepsis. Abnormal total leucocyte count, abnormal PMN count, elevated immature to total (I: T) PMN ratio, immature to mature(I:M) PMN ratio, platelet $\operatorname{count}(<150,000 /$ $\mathrm{mm} 3$ ) and degenerative changes in PMNs. An abnormal total PMN count is assigned score of two instead of 1 , if no mature polymorphs are seen on the peripheral smear to compensate for the low I:M ratio. Score of two and $<2$ was interpreted as sepsis is unlikely, score 3-4 asepsis is possible and score 5 and above as sepsis is very likely.

C-reactive protein levels were also recorded from Immunology laboratory and quantitative evaluation of CRP done.

Blood samples for culture and sensitivity was sent from NICU to Department of Microbiology in specifically provided sterile blood culture bottles and reports obtained after 72 hours. The diagnosis of sepsis was confirmed when there were positive findings in blood culture.

\section{Data Analysis}

Data analysis was carried out in Microsoft excel. Data was filtered, coded and analysed using Chi-square test for quantitative variables in SPSS version 16 (Statistical Package for Social Sciences). In all the tests p-value of less than 0.05 was taken to be statistically significant and values less than 0.01 was taken highly significant.

\section{Result}

Among the 80 cases studied, 12 cases indicated a positive culture while the remaining $85 \%$ indicated a negative culture. The two groups were analyzed for baseline parameters, bacteriological Profile and hematologic Parameters (Table1,2).

Males predominated females (3:1) in the culture-positive group. Late-onset sepsis (more than 72 hours) was more frequent (58\%). There was no significant impact of birth weight with both groups being represented equally. Preterm babies were under-represented in the study $(9 / 80)$ and thus contributed to only $33 \%$ of the culture-positive cases.

The most common causative organism in the study were E. coli and Staphylococcus aureus (Fig 4) each of which attributed to $25 \%$ followed by Streptococcus pneumonia and Group B beta Streptococci (GBS) which constituted $16 \%$ each.

Abnormal TLC (Leukocytosis/Leukopenia) was seen in $67 \%$ of the culture-proven cases of which $>50 \%$ showed leukocytosis (Fig. 1) and the remaining indicated leucopenia. $34 \%$ of culture-positive babies had a normal leukocyte count.

Absolute Neutrophil Count $(<2000)$ was seen in $66 \%$ of the culture- positive cases whereas it was seen only in $6 \%$ of the culture-negative group.

Immature Neutrophil Count- 75\% culture positive cases had an elevated immature neutrophil count.

Both I:T ( $<0.2)$ and I:M ratio $(>0.3)$ were significantly associated with culture positive group. (P-value $<.002$ )

Degenerative changes All the culture positive cases showed degenerative changes in neutrophils. The most common degenerative changes seen were toxic granulations followed by cytoplasmic vacuolations (Fig. 2).

Platelet Count- None of the culture-positive cases showed platelet count $<1.5 \mathrm{~L}$ but $8 \%$ of culture-negative neonates exhibited thrombocytopenia (Fig. 3)

C Reactive Protein- Only 9\% of culture positive cases gave nonreactive CRP and found to be statistically significant (p-value 0.011).

HSS- $41 \%$ of culture-positive neonates have a Hematological score of $>5$, which is suggestive of sepsis. $33 \%$ of culture- positive shows a score which indicates probable sepsis and $26 \%$ cases gives a score suggesting that sepsis is unlikely. Only $7 \%$ of culture-negative cases indicated a hematological score suggestive of sepsis.

\section{Discussion}

Neonatal sepsis is a serious illness with high morbidity as well as mortality. Early diagnosis with prompt antibiotic therapy can significantly improve the outcome. So, a 
Table 1: Hematological profile.

\begin{tabular}{|l|l|l|l|}
\hline Parameter & $\begin{array}{l}\text { Culture Positive } \\
(\mathbf{N}=12)\end{array}$ & $\begin{array}{l}\text { Culture Negative } \\
\mathbf{N}=(68)\end{array}$ & $\mathbf{p}$ Value $<0.05$ \\
\hline Abnormal TLC & $8(67 \%)$ & $11(16 \%)$ & YES \\
\hline ANC $<2000$ & $8(67 \%)$ & $4(06 \%)$ & YES \\
\hline Increased immature PMN & $9(75 \%)$ & $11(16 \%)$ & YES \\
\hline I:T $>0.2$ & $9(75 \%)$ & $13(19 \%)$ & YES \\
\hline I:M $>0.3$ & $7(58 \%)$ & $9(13 \%)$ & YES \\
\hline Degenerative changes & $12(100 \%)$ & $44(65 \%)$ & YES \\
\hline Platelet $<1,50,000 / c u . m m$ & $0(0 \%)$ & $6(9 \%)$ & NO \\
\hline CRP -reactive & $11(92 \%)$ & $35(51 \%)$ & YES \\
\hline H.S.S $\geq 3$ & $9(75 \%)$ & $20(29 \%)$ & YES \\
\hline
\end{tabular}

Table 2: Statistical analysis of hematologic parameters.

\begin{tabular}{|l|l|l|l|l|}
\hline Parameter & Sensitivity & Specificity & PPV & NPV \\
\hline Abnormal Leukocyte Count & $66 \%$ & $83 \%$ & $42 \%$ & $93 \%$ \\
\hline ANC & $66 \%$ & $94 \%$ & $66 \%$ & $94 \%$ \\
\hline INC & $75 \%$ & $83 \%$ & $45 \%$ & $95 \%$ \\
\hline I:T & $40 \%$ & $94 \%$ & $75 \%$ & $80 \%$ \\
\hline I:M & $55 \%$ & $86 \%$ & $46 \%$ & $92 \%$ \\
\hline Degenerative change & $100 \%$ & $35 \%$ & $21 \%$ & $100 \%$ \\
\hline Thrombo-cytopenia & $0 \%$ & $91 \%$ & $84 \%$ \\
\hline CRP & $91 \%$ & $48 \%$ & $23 \%$ & $97 \%$ \\
\hline HSS & 41 & 92 & 50 & 90 \\
\hline
\end{tabular}

Table 3: Comparing degenerative changes in present study with other studies.

\begin{tabular}{|l|l|l|l|l|}
\hline Author & Sensitivity & Specificity & PPV & NPV \\
\hline Narasimha A (2011) & $68.4 \%$ & $66.6 \%$ & $66.6 \%$ & 40 \\
\hline Makkar M (2013) & $78.1 \%$ & $94.4 \%$ & $92.5 \%$ & $82.9 \%$ \\
\hline Supreetha (2015) & $53 \%$ & $89 \%$ & $68 \%$ & $82 \%$ \\
\hline Present & $100 \%$ & $35 \%$ & $21 \%$ & $100 \%$ \\
\hline
\end{tabular}

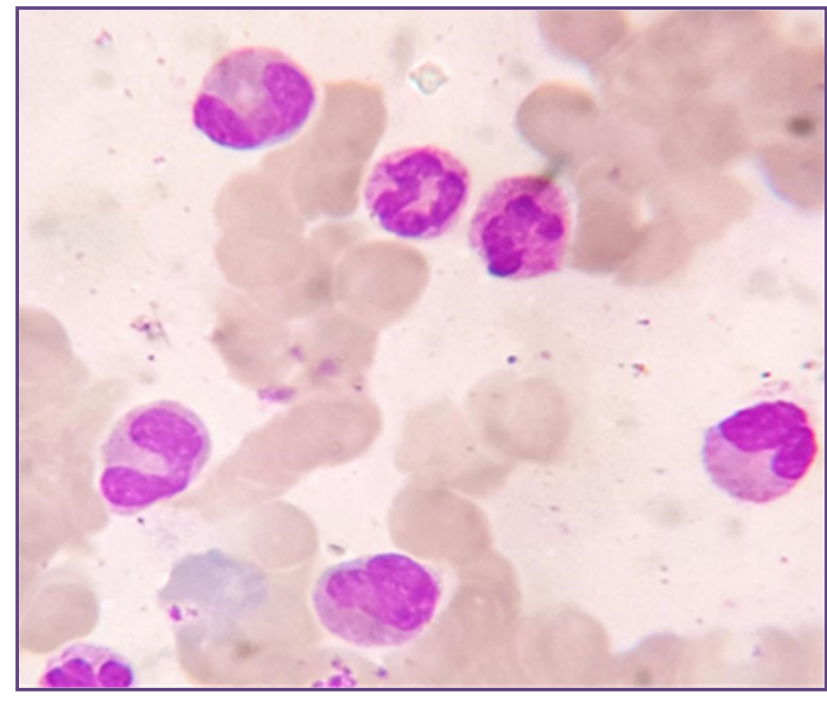

Fig. 1: Peripheral blood film showing increased number of immature neutrophils (Leishman stain; X1000).

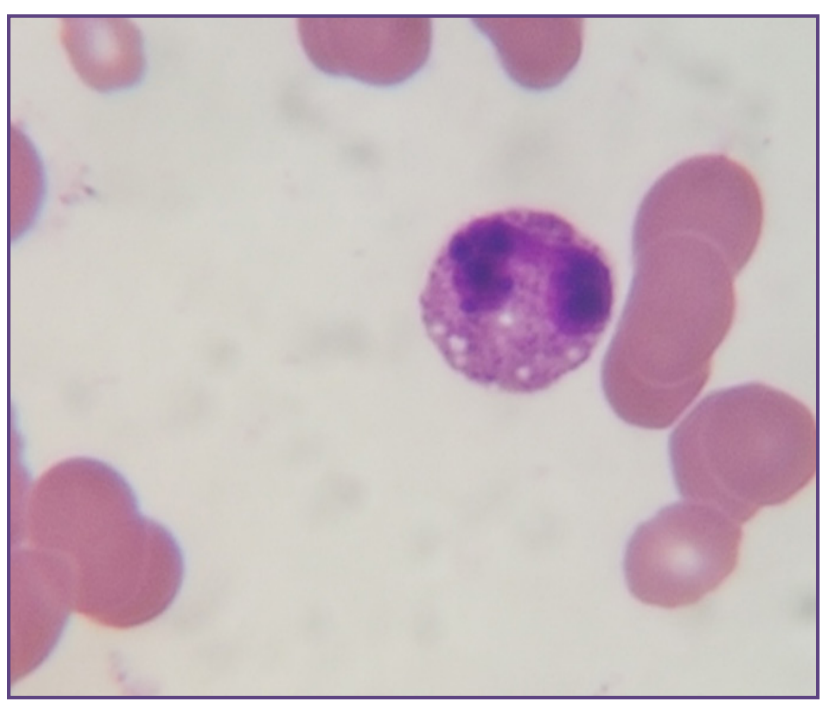

Fig. 2: Peripheral blood film showing Cytoplasmic vacuolation in neutrophil (Leishman stain; X1000). 


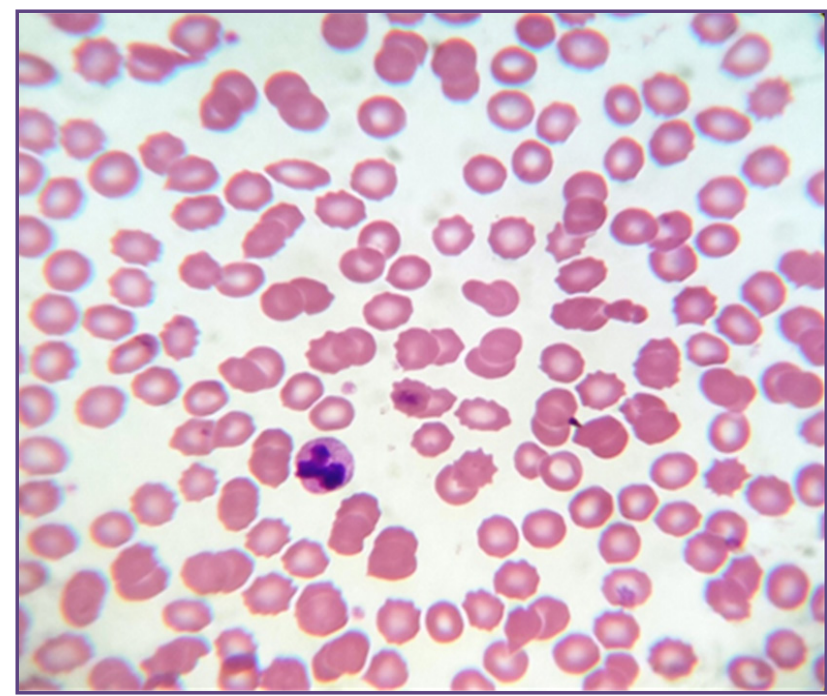

Fig. 3: Peripheral blood film showing thrombocytopenia (Leishmans stain; X100).

screening test is necessary for the detection and treatment of neonatal sepsis. Blood culture which is considered the gold standard has very low sensitivity due to pre- and post-analytic factors and is also not available within the therapeutic window ${ }^{[9]}$. Our study showed a culture positivity of $12 \%$ which is similar to other studies. ${ }^{[5]}$. The culturepositive cases were correlated with various hematologic parameters, CRP reactivity and HSS of Rodwell et al ${ }^{[8]}$ to assess the sensitivity, specificity PPV, NPV and their utility in the early diagnosis of sepsis.

Baseline characteristics: Male gender and low birth weight were more frequently associated with sepsis, similar to studies of Buch et al ${ }^{[10]}$ and Varsha et al ${ }^{[2]}$. This is attributed to factors regulating synthesis of $\square \square$ globulin which is situated on the X-chromosome and the low maternal IgM seen in low birth weight babies as proposed by Buch et al. $\left.{ }^{10}\right]$ Age of onset of sepsis- Among culture-positive cases only $42 \%$ of cases were in the early neonatal period (within 72 hours). This correlates with the study by Nandy et al ${ }^{[11]}$ (48 hrs. or $72 \mathrm{hrs}$.). Late onset sepsis occurs due to low birth weight, prematurity, admission in intensive care unit, mechanical ventilation, invasive procedures and administration of parenteral fluids. ${ }^{[12]}$

Pre-term infants were underrepresented in our study $(12 \%)$ $33 \%$ of whom were culture positive comparable to the studies done by Sriram et $\mathrm{al}^{1}$ and Supreetha ${ }^{[3]}$.

Hematological parameters- Among the hematologic parameters Immature Neutrophil Count was the best performing index with high sensitivity, specificity and NPV, similar to studies by Makkar et $\mathrm{al}^{9}$ and Khair et al ${ }^{[13]}$. Degenerative changes and CRP were highly sensitive

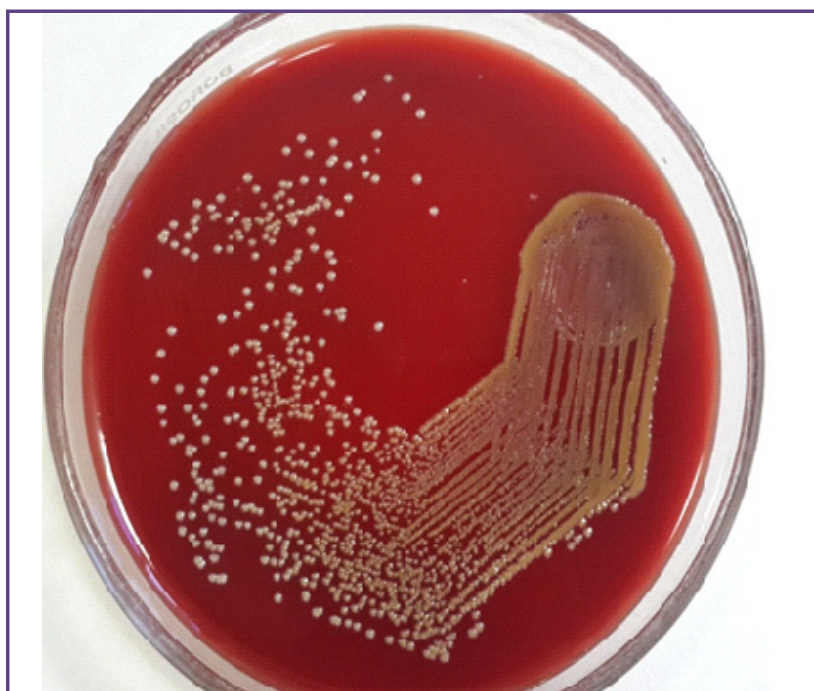

Fig. 4: Staphylococcus aureus grown on blood agar as golden yellow colonies

but lacked specificity as they were seen in culture negative cases too. I:T $>0.2$ and IM $>0.3$ were highly specific $(94 \%$ and $86 \%$ respectively) with a high NPV $(95 \%$ and $80 \%)$ similar to studies of Narasimha et al ${ }^{[14]}$ Nandy et al ${ }^{[1]}$ and Makkar et al ${ }^{[15]}$ respectively. Presence of degenerative changes in PMNs showed stress induced leucopoiesis. ${ }^{[16]}$ Degenerative changes in neutrophils exhibited high sensitivity but lacked specificity for sepsis similar to that of Supreetha et al ${ }^{[3]}$. (table:3) While many studies have seen a positive association between sepsis and thrombocytopenia [15,11] none of the culture positive cases in our study indicated a low platelet count. According to Ghosh et al ${ }^{[17]}$ platelet count in isolation is not a reliable predictor of sepsis since thrombocytopenia is common in first week of life. Moreover, culture positive babies with thrombocytopenia do badly. But in our study since none of the culture positive babies showed thrombocytopenia and responded well to treatment. This indicates that thrombocytopenia has a possible prognostic value in culture positive cases.

Studies by Sriram et al ${ }^{[1]}$ have shown a high NPV with an ANC $<2000$. Present study showed 66\%, 94\%, 66\%, 94\% of sensitivity, specificity, PPV, NPV for an ANC $<2000$. Out of the 12 culture positive cases $41 \%$ of cases had a score had score $\geq 5,33 \%$ of cases had score $3 \& 4$. Thus, with a cut off score of $>2$ HSS was highly specific (92\%) with a NPV of (92\%) indicating a HSS of $<2$ can safely rule out sepsis and avoid unnecessary antibiotic treatment and resultant complications.

\section{Conclusion}

The results of the study indicate that HSS can be reliably and effectively used as a screening test to rule out sepsis 
in a neonate where clinical features often overlap with other conditions due to the non-specific symptoms. This avoids unnecessary administration of antibiotics to unaffected babies. HSS increases the diagnostic accuracy of complete blood count and standardizes its interpretation. Moreover, HSS is a cost effective, easily performed by basic qualification doctors in any rural areas with minimal resources. This will ensure immediate action for children with high index of suspicion of sepsis.

\section{Acknowledgements}

Authors are grateful to Head of Department, Pathology for giving permission to conduct the study.

\section{Funding}

Nil

\section{Competing Interests}

The authors declare that there is no conflict of interest.

\section{References}

1. Sriram R. Correlation of blood culture results with the sepsis score and sepsis screen in the diagnosis of neonatal sepsis. Int J Biol Med Res 2011; 2(1):360-8.

2. Varsha, Rusia U,Sikka M, Faridi MM, Madan N. Validity of haematological parameters in identification of early and late onset neonatal infection. Indian J Pathol Microbiol 2003; 46(4): 565-8

3. MS Supreetha, Alva Sathyavathi. Evaluation of neonatal septicemia using hematological parantelers. International Journal of Recent Scientific Research 2015; 6(2):2775-2778.

4. Zaki Mel-S, el-Sayed H.Evaluation of microbiologic and hematologic parameters and E-selectin as early predictors for outcome of neonatal sepsis.Arch Pathol Lab Med. 2009; 133(8):1291-6.

5. Claus Klingenberg, Rene F Kornelisse. Culture Negative Early Onset Neonatal sepsis-At the crossroad between efficient sepsis care and antimicrobial stewardship. Frontiers in Pediatrics.2018;6:285.
6. Shirazi Haider, Sadia Riaz, Tahir Rida. Role of haematological profile in early diagnosis of neonatal sepsis. Ann Pak Inst Med Sci 2010; 6(3):152-6.

7. Mihir J Bhalodia, Surekha B Hippargi. Role of hematological scoring system in diagnosis of neonatal sepsis. Journal of Clinical Neonatology 2017;6(3):144-147.

8. Rodwell RL, Lesile AL, Tudehope DI. Early diagnosis of neonatal sepsis using a haematological scoring system. J Pediatr 1988; 112(5):761-7.

9. Alonso Zea-Vera, Theresa J Ochoa. Challenges in the diagnosis and management of neonatal sepsis. Journal of Tropical Pediatrics.2015;61(1):1-13.

10. Buch AC, Srivastava V, Kumar H, Jadhav PS. Evaluation of haematological profile in early diagnosis of clinically suspected cases of neonatal sepsis.Int J Basic and applied sciences 2011; 1(1):1-6.

11. Nandy M, Dutta S, Ganguly S, et al. Changing Spectrum of Neonatal Septicemia. The Child and Newborn.2007;11:1

12. DE A, Saraswati DE A, Saraswati K, Gogate A, Raghavan K. C-Reactive protein and buffy coat smear in early diagnosis of childhood septicemia. Indian J Pathol Microbiol1998; 41(1)23-6.

13. Khair K B, Rahman MA, Sultana T,et al. Role of Hematologic Scoring System in Early Diagnosis of Neonatal Septicemia. BSMMU J .2010; 3(2): 62-67.

14. Narasimha A, Harend Narasimha A, Harendra kumar M.L. Significance of hematological scoring system(HSS) in early diagnosis of neonatal sepsis. Indian J Hematol Blood Transfus. 2011; 27(1):14-7.

15. Makkar M, Gupta C, Pathak R, Garg S, et al. Performance evaluation of haematological scoring system in early diagnosis of neonatal sepsis. J Clin Neonatol 2013; 2(1):25-9.

16. Saleem M,Shan Israr K.Hematolgical scoring system for early diagnosis of neonatal sepsis.Journal of Rawalpindi Medical College 2014;18(1):68-72.

17. Ghosh S, Mittal M. Early Diagnosis of Neonatal Sepsis using a Hematological System.Ind J Med Sci 2001;55(9):495-500

*Corresponding author:

Aparna S, Swasti, GHRA45/1, Gaffhills, chekalamukku, Sreekariyam, Trivandrum, Kerala. 695017

Phone: +91 9496366954

Email: aparnasnair.9@gmail.com

Date of Submission : 16/07/2020

Date of Acceptance : 21/09/2020

Financial or other Competing Interests: None.

Date of Publication : 30/10/2020 